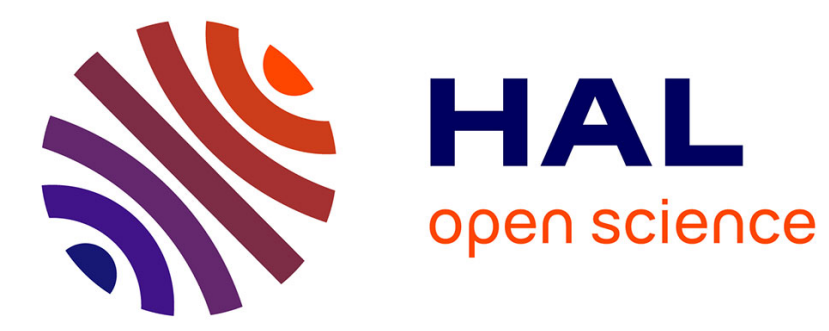

\title{
Toward a topic-specific logicism? Russell's theory of geometry in the Principles of Mathematics
}

Sébastien Gandon

\section{To cite this version:}

Sébastien Gandon. Toward a topic-specific logicism? Russell's theory of geometry in the Principles of Mathematics. Philosophia Mathematica, 2008, 16 (3), pp.1-39. halshs-00326371

\section{HAL Id: halshs-00326371 https://shs.hal.science/halshs-00326371}

Submitted on 17 Oct 2008

HAL is a multi-disciplinary open access archive for the deposit and dissemination of scientific research documents, whether they are published or not. The documents may come from teaching and research institutions in France or abroad, or from public or private research centers.
L'archive ouverte pluridisciplinaire HAL, est destinée au dépôt et à la diffusion de documents scientifiques de niveau recherche, publiés ou non, émanant des établissements d'enseignement et de recherche français ou étrangers, des laboratoires publics ou privés. 


\title{
Toward a topic-specific logicism? Russell's theory of geometry in the Principles of Mathematics.
}

\author{
SÉBASTIEN GANDON \\ PHIER, Université Clermont-Ferrand II, 4 rue Ledru, 63000 Clermont-Ferrand, \\ FRANCE
}

"The tragedy of Russell's paradox was to obscure from Frege and from us the great interest of his actual positive accomplishment".

([Boolos, 1998], p. 267)

\begin{abstract}
Russell's philosophy is rightly described as a programme of reduction of mathematics to logic. Now, the theory of geometry developed in 1903 does not fit this picture well, since it is deeply rooted in the purely synthetic projective approach, which conflicts with all the endeavours to reduce geometry to analytical geometry. The first goal of this paper is to present an overview of this conception. The second aim is more far-reaching. The fact that such a theory of geometry was sustained by Russell compels us to question the meaning of logicism: how it is possible to reconcile Russell's global reductionism standpoint with his local defense of the specifities of geometry?
\end{abstract}

\section{Introduction}

At the turn of the XXth Century, Russell and Poincare confronted each other in two very notorious debates about foundational issues - the first one about geometry (1898-1900), the second about the role of logic in mathematics $(1906-1911)^{1}$. This clash between two major figures of twentieth Century's science and philosophy with time gained the dimension of a mythological battle, which opposed two radically different approaches to mathematics: Poincaré, refusing to found mathematics on any foreign discipline, was seen as the champion of the working mathematicians, while Russell, rejecting any psychological consideration, was regarded as the crusader of a new faith, according to which mathematics, in all its varieties, could be reduced to a few logical premisses.

Of course, that kind of dramaturgy tends to rigidify the two doctrines. Poincaré's intuition was not only a psychological faculty, since it was linked in many ways to some contemporary formal developments ${ }^{2}$ - and, as we will see, Russell's logicism was in many ways also the result of some contemporary changes in mathematical practices. But this appears to be often forgotten, and the mythological figure of the two opposing giants seems to overdetermine the reading of both Poincaré's and Russell's work.

The reception of Russell's philosophy of mathematics has been especially affected by this controversy. Let me quote a very telling letter that the French mathematician J. Dieudonné wrote to P. Dugac (12/05/1984):

The controversy between Poincaré and Russell is very enlightening; it reveals quite obviously how completely invalid the reasonings of the alleged 'mathematician' Russell are about everything connected to mathematics; he should have been wholly

\footnotetext{
${ }^{1}$ For the first polemic, see [Poincaré, 1899], [Poincaré, 1900], and [Russell, 1899b]; for the second one, see [Poincaré, 1906], [Poincaré, 1909] and [Russell, 1906], [Russell, 1910], [Russell, 1911]. [Heinzmann, 1986] presents all the pieces of the second debate.

${ }^{2}$ For a criticism of the psychological reading of Poincaré's intuition, see [McLarty, 1997].
} 
self-taught on the subject, since what he says shows he apparently didn't know anything about the works on the foundation of geometry, from Cayley to Pasch, Klein, and the Italian school [...]. I think that Poincaré was too kind to find time to discuss this verbiage, and to explain the Erlangen Programme again and again (without quoting it, probably so as not to scare the readers of the Revue de Métaphysique et de Morale); this was, however, a waste of effort, since Russell did not understand anything about it, and continued to use the words 'true' and 'false' wildly in connection with mathematics and its relation to reality (words that Poincaré avoided with great care). [...] The moral is: philosophers should know something about mathematics before claiming to speak about it! ${ }^{3}$

Happily, the reactions of Russell's readers are likely to not always be as intense as Dieudonné. But the idea that Russell's work was cut from the ongoing mathematical research of his time is quite widespread among historians of mathematics.

Described in this way, the contrast between Russell's and Poincaré's approach is too vague to be discussed. More recently, M. Detlefsen ([Detlefsen, 1990], [Detlefsen, 1993]) has given an interpretation of the opposition between the two writers which, while encapsulating the core of the suspicion with which Russell's logicism is sometimes looked at by mathematicians, is sufficiently precise to allow a conceptual analysis. Elaborating on a criticism first made by Poincaré, M. Detlefsen points out that:

The inferences of the mathematical reasoner reflect a topic-specific penetration of the subject being reasoned about that is not reflected in the topic-neutral inferences of the logical reasoner. Logical mastery of a set of axioms (...) does not in itself bespeak any significant mathematical insight into the subject thus axiomatized. To use Poincaré's own figure, the logical reasoner is like a writer who is well-versed in grammar but has no ideas for a story. The mathematical reasoner, on the other hand, is guided by his grasp of the 'architecture' of a subject, and his inferences thus move according to a metric determined by this distinctive local 'design'. Sensitivity to this grasp of local architecture is, in Poincaré's view, the key factor separating the mathematician's epistemic condition from that of the logician. ([Detlefsen, 1993], p. 31)

While mathematical knowledge is defined as being topic-specific, logical knowledge is characterised as being always global and topic-neutral ${ }^{4}$, and given the opposition between the two, the logicist endeavour to reduce mathematical to logical knowledge is, according to Detlefsen, a complete failure.

More precisely, the logician seems to be missing the pecularity of the mathematical topic he is talking about in at least two ways. Focusing only on the inferential steps, the logical reasoner is unable to grasp the whole structure of his subject. As Poincaré said:

When the logician shall have broken up each demonstration into a multitude of elementary operations, all correct, he still will not possess the whole reality; this I know not what which makes the unity of the demonstration will completely escape him. [...] Pure logic cannot give us this appreciation of the total effect; this we must ask of intuition. ([Poincaré, 1908]; quoted in [Detlefsen, 1993], p. 32)

But, in another way, the logician looks at his subject from too far away. Indeed, logic is topicneutral: the logical inferential rules apply to any field at all, and thus provide him with no information about the specific subject under investigation. The logical reasoner looks then at his object from too far away or from too close, but never from the right distance. He is always blind to the mathematically relevant structure he claims to grasp.

\footnotetext{
${ }^{3}$ Quoted in [Dugac, 2003], p. 221-222.

${ }^{4}$ Of course, Detlefsen's theory is much richer and more intricated than that - but this way of putting the matter is by itself already sufficiently interesting to start with. The aim of this article is not to discuss Detlefsen approach, but only to discuss the conception of Russell's logicism which is conveyed by this approach.
} 
According to Detlefsen, the fault of the logicist, who aims at blurring the difference between logic and mathematics, is then very severe. If no genuinely mathematical proof can be logicized, it is because, when the proof is successfully enregimented in the logical notation, the insight into the local 'architecture' (the proof initially provided with) is completely lost. For Detlefsen, it is the broad logicist epistemological stance that is then completely misguided; logical standardization, whether possible or not, goes directly against what is needed by a proper mathematical understanding, that is to say against having a grasp of the local design of the subject investigated. Only the 'I know not what' that intuition is, could provide us with the means by which to regain the intelligibility of the mathematical topic.

Detlefsen's stimulating contrast directly leads to the very core of the issue I would like to raise. Since it is traditionally regarded as the field where intuition and insight play the most important role, geometry is, if anything, a topic-specific mathematical subject. On the other hand, Russell's work is explicitly designated as the proximate target of both Detlefsen's and Poincaré's attacks. Now, Part VI of The Principles of Mathematics is entirely devoted to geometry. If Detlefsen were right, Russell's logical approach would lead to breaking and dissolving the essential unity of geometry - Russell's logicized geometry would no longer be a proper geometry. But is this really the case? Is it true that Russell's logicism could not provide us with a characterisation of geometry as a particular mathematical field?

To anticipate my analysis, it will appear that if Detlefsen was right to emphasize the need for having a topic-specific approach of mathematics, the picture he drew of Russell's programme is flawed. As we will see, Russell's logicist theory of geometry was topic-specific. What is more, Russell not only developed a very sophisticated theory of geometry, but he did so with the explicit aim of contrasting it from mathematical analysis - that is, with the explicit aim of articulating his own topic-specific penetration of the subject.

This point is crucial in terms of interpreting what is at stake in the logicist project. Remembering the opposition between Poincaré and Russell, one would probably expect that the logicist answer to Detlefsen's stance would proceed according to the following lines: the distinction between mathematical (topic-specific) and logical (topic-neutral) knowledge is a mere psychological distinction, which has nothing to do with the content of any mathematical proposition. Thus, even if it could have a bearing on some epistemological issues, it should be, as such, neglected by any theory which, like logicism, aims at accounting for the 'true' content (the logical and not the psychological content) of mathematics. But, in fact, the answer we could extract from the Principles does not take this quite unexciting shape: Russell's logical theory of geometry was topic-specific. And this raises a certain number of questions for the on-going interpretations of Russell's logicism as a whole.

Indeed, all the various readings of Russell's works seem to regard arithmetic as the place where logic and mathematics meet each other and interact ${ }^{5}$. Thanks to the works of Cantor and Dedekind, and to the development of the so-called 'arithmetization of mathematics', once the theory of progression is derived from logic, nothing really fundamental remains to be done. The arithmetical definition of continuity allows us to develop the differential calculus, and at the same time, (analytic) geometry. In order to grasp the meaning of Russell's logicism, one could think, then, that it would be enough to focus on part II of the Principles which is devoted to whole numbers. The rest of the book, even if mathematically interesting, would teach us nothing new about the articulation between mathematics and logic ${ }^{6}$.

The problem is that Russell's theory of geometry in the Principles does not fit this schema at all. The simple fact is that Russell does not regard geometry as an extension of analysis. It seems thus that, when trying to uncover the meaning of Russell's logicism, it is not sufficient to focus only on the arithmetical case. Of course, the mere fact that Russell did not regard geometry as an extension of arithmetic does not mean that the various interpretations of logicism we found in the secondary litterature (all based on Russell's theory of whole numbers) are false. It

\footnotetext{
${ }^{5}$ Scholars as different as P. Hylton, G. Landini and B. Linsky agree on this point; see [Hylton, 1990], p. 187-189; [Landini, 1998], p. 16-21; [Linsky, 1999], p. 131-132.

${ }^{6}$ I discuss Russell's view about arithmetization in [Gandon, 2008b].
} 
nevertheless compels us to shift, at least for a short while, the perspective. Among the different interpretations of Russell's logicism, which one can account for the theory of geometry Russell actually developed? There seems to be a tension between Russell's global logicist reductionism and the sensitivity he displayed for the peculiarity of the geometrical sciences. How can we account for this fact? Should we really take for granted that Russell's logicism was, as Detlefsen has thought, topic-neutral? Couldn't we conceive Russell's programme as an endeavour to construct a 'topic-specific logicism'?

In the sequel, I will not properly adress these questions. Indeed, I would rather like to focus on Russell's neglected theory of geometry to show that it does not fit into the frame set by Detlefsen. My aim here will be to draw attention to the fact that something philosophically significant is going on after part II of the Principles, and that this fact might raise a problem for the way we view Russell's achievement today. My goal in this article will thus not be to solve a problem, but to elaborate on a question. What follows is not a new interpretation of Russell's logicism, but it could be taken as presenting a challenge to the current readings of the Principles.

Three sections will divide my argument. I will first expose (section 2) the mature theory of geometry found in the Principles. I will contend that Russell defined geometry as a theory of incidence relations - and not as a theory of order. Secondly, I will explain (section 3) the reason behind this surprising dismissal of ordinal relations. As the reading of his former texts on geometry shows, the young Russell was a follower of the XIXth century's synthetic geometrical tradition. Russell still stuck to this line of thought in 1903. The idea that geometry was nothing other than a science of incidence (and not of order) was the upshot of his prior commitments. In the final part (section 4), I will show that Russell's definition of geometry in terms of incidence fits well with some contemporary researche initiated by such mathematicians as Moore and Veblen which means that one cannot dismiss Russell's conception as being just an abstract philosophical view of geometry completely severed from the actual developments in 'real' mathematics. Having established that Russell's theory of geometry was topic-specific, I will briefly come back, in my conclusion, to the philosophical meaning of this fact: how can we reconcile Russell's reductionist global logicist stance with the local features of Russell's account of geometry?

\section{Russell's conception of geometry in The Principles.}

Part VI of [Russell, 1903], which is entirely devoted to geometry, is quite sizeable, since it filled nearly one hundred pages of the book. It could itself be subdivided into three sections. The first one, which comprises the three first chapters, was devoted to projective geometry ${ }^{7}$; the second one, which included the following three chapters, dealt with metrical geometry; the last three chapters of the Part were dedicated to some philosophical issues connected with the notion of space. I will completely leave aside this last topic in the sequel, to focus only on the two first sections. How can we explain this opening distinction between projective and metrical geometry?

In his first book, An Essay on the Foundations of Geometry, Russell, following Klein ${ }^{8}$, explained how the three classical metrical three-dimensional geometries (the Euclidean, the Hyperbolic and the Elliptic) could be developed in a projective setting. The method was rather complicated, but the mathematical reasoning was widely known and used at the time ${ }^{9}$. As we

\footnotetext{
${ }^{7}$ The projective geometry is basically a geometry dealing with incidence and order between points, lines and planes, with the exclusion of all metrical considerations. If no notion of distance occurs in it, a relation between two pairs of collinear points, the anharmonic ratio, is invariant by projective transformation; for more on the topic, see below and [Coxeter, 1949]. Projective geometry was developed in the XIXth Century and was taken, especially in England, as a means to salvage the unity of geometry from the dangers of fragmentation caused by the arising of the non-metrical geometries; see [Richards, 1988].

${ }^{8}$ There is a more than 100 folio manuscript devoted to Klein's presentation of geometry kept by the Russell Archives at McMaster

${ }^{9}$ In a series of articles published in the Mathematische Annalen from 1871 to 1874 , Klein showed how to derive the various metrical geometries from the projective geometry. These papers created a considerable stir in the mathematical public, because they put for the first time non-euclidian geometries on the same footing as Euclidian geometry: they were all regarded as an outgrowth of the same all-encompassing projective theory. In the first of these papers ([Klein, 1871]), Klein showed how to define the various linear and angular distances
} 
shall see below, Russell did not endorse this reduction of all metrical concepts to projective ones, but he clearly assumed that metrical geometry presupposed the projective frame. For Russell then, defining the nature of geometry was thus, ultimately, defining the nature of projective geometry.

Russell did not change his mind in 1903: the first part of [Russell, 1903], Part VI, in which he tried to define what geometry was, came prior to the second one, which was devoted to the analysis of metrical or, as he sometimes says, quantitative geometry. In order to grasp Russell's mature conception of geometry, we have to focus then, primarily, on the three first chapters 44-46. I will leave the first one aside for the moment. Now, chapter 45 and chapter 46 offer two different accounts of what projective geometry is. The second one, inspired by Pasch $^{10}$ (chap. XLVI), started from the ordinal relation 'between'; the first one, inspired by Pieri ${ }^{11}$ (chap. XLV), started from incidence relations. Why did Russell develop two different approaches of projective geometry?

Before answering this question, it is worth looking at a standard presentation. Let us take the classic axiomatic of projective geometry given in [Coxeter, 1947] (p. 20-22). Here, one finds two groups of postulates. First, seven axioms of incidence:

There are at least two points

Any two points are incident with just one line

The line $A B$ is incident with at least one point besides $A$ and $B$

There is at least one point not incident with the line $A B$

If $A, B, C$ are three non-collinear points, and $D$ is a point on $B C$ distinct from $B$ and $C$, while $E$ is a point on $C A$ distinct from $C$ and $A$, then there is a point $F$ on $A B$ such that $D, E, F$ are collinear

There is at least one point not in the plane $A B C$

Any two planes intersect in a line

Then, six axioms of order (the last one is singled out by Coxeter as an axiom of continuity):

If $A, B, C$ are three collinear points, there is at least one point $D$ such that $A B$ separates $C D$

If $A B$ separates $C D$, then $A, B, C, D$ are collinear and distinct

If $A B$ separates $C D$, then $A B$ separates $D C$

If $A, B, C, D$ are four collinear points, then either $A B$ separates $C D$ or $A C$ separates $B D$ or $A D$ separates $B C$

If $A B$ separates $C D$ and $A D$ separates $B X$, then $A B$ separates $C X$

If $A, B, C$ are three collinear points, we define the segment $A B / C$ as the class of points $X$ for which $A B$ separates $C X$

For every partition of all the points of a segment ${ }^{12}$ into two non-vacuous sets, such that no point of either lies between two points of the other, there is a point of one set which lies between every other point of that set and every point of the other set

from the projective anharmonic ratio and from the selection of a quadric surface. For example, if the Absolute were a ruled quadric, the distance between two points $A$ and $B$ (inside the surface) would be the logarithm of the anharmonic ratio of $A$ and $B$ with the two intersections between the line $(A B)$ and the ruled quadric - and the metric would be hyperbolic. Klein claimed, at the end of his first paper, to have reduced the main metrical concepts to the projective ones. And in his subsequent articles, he tried to show that projective geometry itself could be developed without referring to numerical distances or any other metrical concepts. For more on this topic, see [Toretti, 1978].

${ }^{10}$ See [Pasch, 1882] - Russell referred as well to the version that Peano gave to Pasch's work in [Peano, 1894].

${ }^{11}$ See [Pieri, 1898].

${ }^{12}$ The definition of a segment is the following: If $A, B, C$ are three collinear points, we define the segment $A B / C$ as the class of points $X$ for which $A B$ separates $C X$. 
In this presentation, projective geometry appears to be a combination of two subtheories: on the one hand, a theory of the incidence relations between points, straight lines and planes; on the other, a theory of the ordinal relation of separation between pairs of points on a line. With only the incidence axioms, many projective theorems can be proved. For instance, Desargue's theorem is a direct consequence of the postulates of incidence. Many projective concepts can also be defined in terms of incidence relations alone. Thus, the notorious von Staudt's quadrilateral construction gives the means to define the harmonic conjugate of a given point with respect to one given pair of points (see figure 1). This construction uses only the straightedge, and does not refer to the ordinal configuration of the points $A, B$ and $C^{13}$. From Desargue's theorem, it follows that the position of the fourth harmonic $D$ does not depend on the position of the two pairs of lines intersecting in $A$ and in $B$ (Russell calls this result the v. Staudt's unicity theorem).

\section{Figure 1.}

However, the axioms of incidence do not suffice by themselves in order to derive the whole body of the projective geometry. The so called 'fundamental theorem of projective geometry', which says that a projective transformation between two ranges of points is uniquely determined when we are given three points of one and the corresponding three points of the other, along with Pappus's theorem and some others fundamental results, cannot be demonstrated with this equipment. Order axioms, or some equivalent extensions, are essential, here. As we will soon see, the realization of this fact did not come easily. G. von Staudt believed that some iteration of his quadrilateral construction could be used to prove the fundamental theorem. Klein, in his [Klein, 1873], was the first to have uncovered that von Staudt's proof contained some hidden ordinal assumptions ${ }^{14}$. The mathematician did not succeed right away in filling the gap he discovered, and a fascinating story began here, which would last until the birth of the XXth Century (see below for more on this point). But for the time being, let us remind ourselves that in the standard presentation, projective geometry is not only defined as a theory of incidence, but as a theory of incidence and order.

Let me come back to Russell. To oversimplify the facts without distorting them, I could say that, in chapter 46 of the Principles, Russell's approach consisted of presenting projective geometry as a theory of order, and that his first account in chapter 45 amounted to reducing it to a theory of incidence relations. Indeed, Pasch's approach consisted of defining projective geometry in terms of order relations, and Pieri's account amounted to deriving projective geometry from incidence structure alone. In other words, the two axiomatic expositions not only differed in some insignificant formal details; on the contrary, they displayed two completely different insights into what projective geometry was and what the specificity of geometry was in general. This point is crucial. Russell's discussion of geometry did not concern the formal garment one should put on a common theoretical body, but the body itself; it concerned the specificity and the nature of geometrical thought. The two axiomatizations of projective geometry, far from being some unimportant variations on a common topic, carried then two very distinct views of the specificity of geometry. In the sequel, I will try to show, firstly, that chapter 45 and 46 of [Russell, 1903]

\footnotetext{
${ }^{13}$ Let us call a figure composed of four points $P, Q, R, S$, of which no three are collinear, a quadrangle - let us call $P Q R S$ the vertices and the lines $Q R, P S, R P, Q S, P Q, R S$ the six sides of the complete quadrangle finally, let us name the pairs $Q R$ and $P S, R P$ and $Q S, P Q$ and $R S$, the three opposite sides of the quadrangle. Now, according to the German mathematician G. von Staudt, four collinear points $A, B, C, D$ are said to form an harmonic set if there is a quadrangle of which two opposite sides pass through $A$ and two other opposite sides through $B$, while the remaining sides pass through $C$ and $D$. In this case, $C$ and $D$ are said to be harmonic conjugates of each other with respect to $A$ and $B$; see figure 1 .

${ }^{14} \mathrm{G}$. von Staudt showed that, as soon as three points were given, it was possible, by iterating the quadrilateral construction, to cover the whole straight line with a dense set of points - that is, von Staudt proved that three collinear points define a covering dense set of points (usually called the Möbius net) of the projective line, and thus that the image of every point of this set by a projective transformation (which, by definition, left the anharmonic ratio invariant) was determined as soon as the image of the three original points were given. So far, so good. The trouble was that, from this, the mathematician misleadingly concluded that the fundamental theorem was true. In a modern anachronistic language, G. von Staudt confused two distinct ordinal properties: the fact for an ordered subset of the line to be dense, and the fact for the same kind of subset to be dense on the line. For more on this topic, see section 4 below; on G. von Staudt, see [Freudenthal, 1974].
} 
presented two ways one could define the essence of geometry, and secondly, that Russell favoured Pieri's account for providing us with a more fundamental concept.

Let me begin with the theory inspired by Pasch, that Russell labelled 'Descriptive geometry'. This geometry could be informally described as the retriction of the real projective geometry to a convex region, or as high school geometry with congruence and parallelism (in this, it differs from affine geometry) left out. On the topic, Coxeter said (ibid. p. 160):

The $[\ldots]$ descriptive geometry is of theoretical as well as practical interest, since the loss of the principle of duality is compensated by the fact that two points now determine a unique segment. Consequently the relations of incidence and separation, instead of being undefined, are both expressible in terms of the single relation of 'three points' order, or intermediacy.

To show what is meant by defining incidence from the relation between, I have listed the nine axioms and the definitions first formulated by Veblen (in the version Coxeter gives; I have omitted the continuity axiom):

There are at least three points

If $A$ and $B$ are two points, there is at least one point $C$ such that $B$ lies between $A$ and $C$ (hereafter noted $[A B C]$ )

If $[A B C]$, then $A$ and $C$ are distinct

If $[A B C]$, then $[C B A]$ but not $[B C A]$

Definition: the interval $A B$ is the class of points $X$ such that $[A X B]$ with the points A and $\mathrm{B}$; the ray $A / B$ is the class of points $Y$ such that $[B A Y]$; the line $A B$ consists of the interval $[\mathrm{AB}]$ with the rays $A / B$ and $B / A$

If $C$ and $D$ are two points on the line $A B$, then $A$ is on the line $C D$

There is at least one point not on the line $A B$

If $A, B, C$ are three non-collinear points, and $D$ and $E$ are such that $[B C D]$ and $[C E A]$, then there is a point $F$ on the line $D E$ with $[A F B]$

Definition: the plane $A B C$ is the class of points collinear with the pairs of points on the intervals $[B C],[C A],[A B]$.

There is at least one point not in the plane $A B C$

Two planes which have one common point have another ([Coxeter, 1947], p. 161-162)

This axiomatic is very close from Peano's version ([Peano, 1894]) of Pasch's first presentation $\left([\text { Pasch, 1882] })^{15}\right.$. It is roughly the same as the ones given by Russell ${ }^{16}$. As we can see, the geometry exposed here contains only two kinds of indefinables: the points and the betweenness relation; all the other concepts (lines, planes, incidence relations between lines, etc...) are defined in terms of these two. Coxeter is thus perfectly right to say that, in this framework, the incidence relation and the separation are derived from the sole indefinable relation of betweenness.

Of course, the descriptive geometry is distinct from the projective one. But in his great work, Pasch, extending the notion of points, lines and planes, and redefining accordingly the ordinal relations between these elements, showed how to derive all the projective theorems. The procedure is similar, if more complicated, to today's standard projective completion of a given affine space. As the technical details do not concern us, I will not deal with them here ${ }^{17}$. From our perspective, the essential point is that, in chapter 46 of the Principles, Russell based the whole

\footnotetext{
${ }^{15}$ In Pasch initial presentation, the (limited) plane was an indefinable. For more details on Pasch's and Peano's works, see [Gandon, 2008a] .

${ }^{16}$ Instead of starting with the three-termed relation 'between', Russell uses transitive asymetrical relations. This change does not greatly affect the content of the axiomatic - see [Russell, 1903], p. 395.

${ }^{17}$ [Russell, 1903] p. 400-403 provides with a good summary of Pasch's method.
} 
projective geometry on two indefinables: points and betweenness relations - without referring either to incidence or to separation.

Seen from a distance, this approach should suit Russell very much. Indeed, betweenness relations, or equivalently, transitive and asymetrical relations ${ }^{18}$, were a key concept in Russell's overall logicist project. Part IV of his book was entirely devoted to this type of relation, which he explicitly presented as a fundamental one for all mathematics ${ }^{19}$. Pasch's reasoning could have been taken as a dazzling confirmation of this claim: order would be the keystone not only of mathematical analysis, but also of geometry. As suprising as it might appear, we will soon see, however, that Russell would let the opportunity slip.

Let me now turn to chapter 45, and to Russell's other axiomatic. There, Russell relied entirely on the work of the Italian mathematician Mario Pieri ${ }^{20}$. What were the distinctive features of Pieri's reasoning?

In Coxeter's axiomatic presentation of projective geometry, one can easily prove that $a, b$ do not separate $c, d$ if, and only if, there are two points $m, n$ such that $a, b$ and $c, d$ both are harmonic conjugates with respect to $m$ and $n$. That $a, b$ are harmonic conjugates of $m, n$ was noted in the Principles $a H_{m n} b$; furthemore, Russell defined the projective segment $(a c b)$ as the locus of the point $x$ such that $\neg(a c b x)$, i.e. such that $a, b$ do not separate $c, x$ (see figure 2 for a geometrical intuitive interpretation of these expressions). With this notation, the theorem ran as follow:

$$
d \in(a c b) \Longleftrightarrow \exists m \exists n\left(a H_{m n} b \wedge c H_{m n} d\right) .
$$

In the left side of the equivalence, we have an ordinal concept; in the right side, a reference to the harmonic conjugation, which could be explained in terms of the quadrilateral construction, that is, in terms of incidence relations alone. This theorem, sometimes called Darboux's lemma, seems therefore to throw a bridge across the two groups of axiom.

Figure 2.

Pieri's great idea was to take advantage of this fact in order to elaborate a definition of the projective segment (the left side of the equivalence) in terms of the harmonic conjugation (its right side). More precisely, Pieri ([Pieri, 1898], p. 24-29) defined (acb) as the locus of the harmonic conjugate of $c$ with respect to a variable pair of distinct points that are harmonic conjugates with respect to $a$ and $b$. As the quantificational structure of the definition is quite complicated (since it is of the type $\forall \forall \forall \forall \exists \exists$ ), I have given a translation of the definition of the separation in the Russellian notation:

$$
\forall x \forall y \forall x^{\prime} \forall y^{\prime}\left(y^{\prime} \in\left(x y x^{\prime}\right) \Leftrightarrow \exists z \exists z^{\prime}\left(x H_{z z^{\prime}} y \wedge x^{\prime} H_{z z^{\prime}} y^{\prime}\right)\right) .
$$

Thus, the fact that a point belongs to a projective segment, or the fact that two pairs of collinear points to be separated, are both reduced to the fact that there are certain points with respect to which some other points are conjugated - which is a pure incidence theoretical fact, concerning how certain lines intersect in the plane. Russell explained the move quite clearly ([Russell, 1903], p. 386):

Given any three different points $a, b, c$ on a line, consider the class of points $x$ such that $a$ and $c, b$ and $x$ are each harmonic conjugates with respect to some pair of points $y, y^{\prime}(\ldots)$. Here $y, y^{\prime}$ are supposed variable : that is, if any such points can be found, $x$ is to belong to the class considered. This class contains the point $b$, but not $a$ or $c$. Let us call it the segment $(a b c)$. Let us denote the relation of $b$ to $x$ ( $a$ and $c$ being fixed) by $b Q_{a c} x$. Then $Q_{a c}$ is symmetrical, and also $b Q_{a c} x$ implies $a Q_{b x} c{ }^{21}$

\footnotetext{
${ }^{18}$ On this equivalence, see [Russell, 1903], p. 207-214.

${ }^{19}$ [Russell, 1903], p. 199 , p. 226

${ }^{20}$ [Pieri, 1898].

${ }^{21}$ See as well this passage, perhaps even clearer ([Russell, 1903], p. 385): 'If four points $x, y, x{ }^{\prime}, y^{\prime}$ be given, it may or may not happen that there exist two points $a, b$ such that $x H_{a b} y$ and $x^{\prime} H_{a b} y^{\prime}$. The possibility of finding
} 
Here $Q_{a c}$ represents the relation of non separation with respect to $a$ and $c$.

To summarize: in the standard presentation, projective geometry arises from a combination of two different axioms groups, an incidence and an ordinal one. In Pieri's approach, projective geometry is derived from only one indefinable relation, the incidence relation. This is truly beautiful: the projective order on a line is derived from the way the lines intersect in the plane ${ }^{22}$.

Things are a bit more complicated, however. As we have shown, the axioms of incidence are not enough to derive the whole body of projective geometry. So, even if no primitive order relation, and then no axioms of order, are introduced, something else must be given to do the job done by the ordinal postulates in the standard presentation. Pieri showed that three further axioms are needed to endow a segment with all the usual ordinal relations. I have given here the Russellian version of these three postulates ([Russell, 1903], p. 386):

(1) If $d$ is on the line $a b$, but does not belong to the segment (abc), and does not coincide with $a$ or with $c$, then $d$ must belong to the segment $(b c a)$. (...)

(2) If $a, b, c$ be distinct points on a line, and $d$ be a point belonging to both the segments $(b c a)$ and $(c a b)$, then $d$ cannot belong to the segment $(a b c)$. (...)

(3) If $a, b, c$ be distinct collinear points, and $d$ a point, other than $b$, of the segment $(a b c)$.

In spite of appearances, these postulates are not about segments or any other ordinal ideas, and their addition does not thus introduce any new indefinable. Indeed, using the definition of the segment in terms of incidence relations, the three axioms can be reformulated so as to be free of all references to ordinal notions. Yet, they allowed Pieri to use his derived ordinal notion of segment exactly as it is used in the standard presentation, that is as if order were to have been a primitive notion. Pieri completed his axiomatic by a continuity axiom (which did not contain any reference to ordinal notions $)^{23}$, and then succeeded to demonstrate the fundamental theorem of projective geometry.

The last complication does not then change our first conclusion. Russell was right: even if Pieri introduced some new axioms, he nevertheless managed to reduce all the former indefinable relations to the sole incidence. As Coxeter explained ([Coxeter, 1949], p. 33): 'The theorem [according to which $a, b$ do not separate $c, d$ if, and only if, there are two points $m, n$ such that $a H_{m n} b$ and $\left.c H_{m n} d\right]$ is especially significant since it would enable us to define separation in terms of incidence, instead of taking separation to be a second undefined relation. (...) This idea is due to Pieri, [who] reduced the undefined relations to incidence alone and reduced the axioms of order to the [three quoted above].'

I would like to pause a bit on Pieri's intellectual achievement. It would seem at first that there is an insuperable intuitive and conceptual gap between ordinal and incidence notions. The way the points can be disposed on a line seems indeed to be very different from the way points and lines intersect on a plane. Pieri's beautiful result proves that it is not the case - it shows that, for ordering the points on a line, one is only required to set appropriate constraints on the way lines intersect in the plane. This idea is properly breathtaking: what, indeed, seems to be more ultimate than order? When Kant sought to prove that space was irreductibly phenomenal, he told us about the ordinal distinction between the right and the left. This shows that such an ordinal distinction seemed to him to be very basic, very foreign to all other conceptual

such points $a, b$ constitutes a certain relation of $x, y$ to $x^{\prime}, y^{\prime}$. [...] Pieri has shown how, by means of certain axioms, this relation of four terms may be used to divide the straight line into the two segments with respect to any two of its points, and to generate an order of all the points on a line.'

${ }^{22}$ For more on Pieri's achievement, see the very enlightening [Marchisotto, 1995] and [Marchisotto, 2006].

${ }^{23}$ Russell followed the Italian mathematician and stated a continuity axiom à la Dedekind, using only projective segments ([Russell, 1903], p. 387):

If any segment $(a b c)$ be divided into two parts $h$ and $k$, such that, with regard to the order $a b c$, every point of $h$ precedes every point of $k$, while $h$ and $k$ each contain at least one point, then there must be in $(a b c)$ at least one point $x$ such that every point of $(a b c)$ which precedes $x$ belong to $h$, and every point of $(a b c)$ which follows $x$ belongs to $k$.

From this and the other postulates - which, once again, involved only one indefinable concept, the incidence relation - , the fundamental theorem of projective geometry easily followed. 
differences. Even if, in Hilbert's Grundlagen, order was axiomatized and did no longer pertain to intuition, a similar opinion was expressed: ordinal concepts were also separated here from the incidence and the metrical ones - ordinal notions also occupied here their own intellectual nest. Last, but not least, part IV of the Principles suggests that, for Russell as well, ordinal relations were primitive and ultimate. It was these ideas that Pieri attacked. What he challenged was precisely the intellectual insularity of ordinal notions: order could be regarded as a mere byproduct, which came directly from the structure of the incidence relations. This disappearance of the ordinal postulates in Pieri's axiomatic of the projective geometry has thus a very deep conceptual meaning.

But let me resume the story. Russell gave two accounts of projective geometry. In the first, projective geometry was regarded as a theory of order. In the second, projective geometry appeared to be a theory of incidence. There is absolutely no doubt that Russell took the second account to be the most fundamental. For Russell, projective geometry was a theory of incidence relations, not a theory of order. ${ }^{24}$ Indeed, Russell defined the nature of geometry in chapter 54 (the first chapter of Part VI) as 'the study of series of two or more dimensions' or as the study of multiple series. For the Russell of the Principles, all geometries involved at least a theory of the way in which series intersect each other, that is, they included at least a theory of incidence.

This choice for Pieri against Pasch is extremely surprising. Indeed, not only in his metaphysics but also in his theory of mathematics, Russell placed ordinal relations at the very heart of his thought. Now, we have just seen that Russell technically had the means to construct projective geometry on a purely ordinal basis. So, why did he refuse to do so? Why did he appear in Part VI of the Principles to be willing to avoid order, while he seemed to have, from an overall point of view, all the reasons to put it forward? Let me specify the difficulty by quoting a passage from [Coxeter, 1949] (p. 33):

[Pieri's] simplification is to some extent illusory, as these axioms would be quite complicated if we expressed them directly in terms of incidence. Now, which is preferable : a number of simple axioms involving two undefined relations, or fewer but far more complicated axioms involving only one such a relation? The answer is a matter of taste.

Given the role played by ordinal notions elsewhere in the Principles, how can we explain Russell's taste here?

\section{The historical background of Russell's theory.}

To answer this question, we need to turn to the genesis of Russell's theory. Describing in detail the evolution of Russell's thought from An Essay to the Principles is too big a task to be undertaken here. Nevertheless, since to understand the meaning of Russell's mature theory, this story has to be taken into account, I will here give an overview of a complicated gestation I have described elsewhere in detail ${ }^{25}$. I would like to put forward the coherence of Russell's efforts: his emphasis on incidence relations is the direct result of his initial involvement in von Staudt's tradition of pure geometry.

In An Essay (1898), Russell confronted the analytical approaches of geometry, and sided with the upholders of the purely geometrical methods. He thus explained that the history of metageometry (i.e. non-euclidean geometries) may be divided into three periods: the synthetic, the metrical and the projective. The first period corresponded to the works of Lobatchewsky and Bolyai, who explored axiomatically the consequences of the negation of the axiom of parallels.

\footnotetext{
${ }^{24}$ Let me quote this passage, about which I will have more to say soon ([Russell, 1903], p. 421): 'The true founder of non-quantitative Geometry is von Staudt. It was he who introduced the definition of a harmonic range by means of the quadrilateral construction, and who rendered it possible, by repetitions of this construction, to give projective definitions of all rational anharmonic ratios. [...] But there remained one further step, before projective Geometry could be considered complete, and this step was taken by Pieri.'

${ }^{25}$ See [Gandon, 2004]; on Russell's evolution during the period, see as well [Griffin, 1991].
} 
According to Russell, the 'metric stage', inaugurated by Riemann, had a much deeper import: '[it] regarded space as a particular case of the more general conception of a manifold [and] taking its stand on the methods of analytical metrical Geometry, it established two non-Euclidean systems, the first that of Lobatschewsky, the second (...) a new variety, by analogy called spherical' ([Russell, 1897], p. 18). The last period in which Russell placed himself, 'is chiefly distinguished from the second, in a mathematical point of view, by its method, which is projective instead of metrical' (Ibid.). Russell's aim was to develop the philosophical interpretation of the third approach. In so doing, he was led to contrast the analytical cartesian methods with the 'direct' approaches inherited from the works of the pure geometers like Poncelet, Chasles, Steiner, von Staudt... ${ }^{26}$.

Since these disputes about the status of analytical geometry are nowadays not very well known, a few words on these debates could be useful. After the Cartesian reform, it was common to oppose the ancient geometry of the Greek with the new analytical one. To resolve any given problem, the former used some piecemal ad hoc tricks, where the latter proceeded according to a uniform and general path. What is more, some general theorems, which could be easily demonstrated using numerical coordinates, exceeded the power of the old synthetic approach. It's no surprise, then, if the new analytical geometry was soon to wholly supersed the Greek geometry. At the beginning of the XIXth however, several mathematicians sought to challenge this diagnosis. According to them, one advantage of the ancient method was that it never lost sight of the geometrical aspect of the subject. The mathematical superiority of the analytical method had thus an epistemological cost: the geometrical topic-specific features of the various problems, covered by a numerical veil ('by the hieroglyphs of the analysis', as stated by L. Carnot in [Carnot, 1803], p. 12-13), were completely lost ${ }^{27}$. The new task was then to develop a new pure geometry, that would be able to compete with the analytical methods. All these geometers attempted to supplement the Greek geometry with some general principles, allowing them to recover the strength of the analytical procedures without loosing contact with the geometrical material. This was the explicit aim of Poncelet's 'principe de continuité' or of Chasles's 'principe des relations nécessaires'. Projective geometry was erected in this highly polemical context: it represented the new pure geometry, more general in his method than the old Greek practice, but wholly opposed to the Cartesian numerical approach, which introduced some foreign hieroglyphic elements into the theory of pure space ${ }^{28}$. Russell's distinction of three periods in metageometry resumed this schema: the synthetic approach corresponded to the geometry of Euclid; the metrical method was on the analytic cartesian one; and the projective represented the new pure geometrical conception.

Note that the very terms of the dispute remind us very much of Detlefsen's distinction between topic-neutral and topic-specific knowledge. The upholders of the pure geometrical method criticised their opponents for exactly the same reasons Detlefsen's Poincaré criticised the logicist - they, as analysts, didn't count the topic-specific character of geometry. The idea that the reasoning of the 'Cartesian' was a pure mecanism, blind to every distinctive features of the subject under study ( $i$. e. exactly like the reasoning of the logicians for Detlefsen), pertained to the folklore of this tradition.

As we have just stated, Russell saw himself as a part of this movement. According to him,

\footnotetext{
${ }^{26}$ See especially [Russell, 1897], p. 69-74.

${ }^{27}$ Let me quote a seminal passage of [Carnot, 1803], p. 9: '[Synthesis, i.e. the old geometric method] is restricted by the very nature of its procedure; it can never loose sight of its object: this object should always be exposed to the mind in a neat and genuine way [...]. [Synthesis] then cannot reason about non real quantities, non possible operations; it can make use of some signs, which could help the imagination and memory; however these signs can be nothing but mere abbreviations. Analysis, on the contrary, has, firstly, all the means of synthesis, and, what is more, it allows some objects which do not exist in these combinations; it represents them by some symbols, as if they were real: it mixes the real being with the being of reason; then, by some methodical transformations, it manages to eliminate [...] them. Then, what was unintelligible in the formulae disappears, and there only remains what a subtle synthesis would probably have discovered: but this result has been obtained by a shorter and easier, nearly purely mechanical, way.' Note that, according to Carnot, the fact that the analytical method allows itself to loose sight of its object is the very origin of his mathematical fruitfulness.

${ }^{28}$ Von Staudt's work should as well be seen as a part of this reaction - von Staudt's project was indeed to demonstrate, using only pure geometrical means, how the coordinates could be introduced in a projective setting. See below for more on this point.
} 
the projective geometry logically came before metrical geometry, and this was what was behind his refusal to endorse the riemannian analytical approach. Metrical geometry was described as a theory of quantity, while projective geometry was defined as a doctrine of quality (and for Russell, the notion of quantity presupposed the notion of quality). Now, to characterize metrical geometry as a quantitative theory did not raise any difficulties - but in what sense exactly could projective geometry be defined as a geometry of quality? This assertion was very vague. In the Essay, Russell claimed to have identified the 'postulates' of the projective theory, but the 'axioms' he listed can hardly be taken as genuine axioms, even when assessed according to the contemporary standard ${ }^{29}$. What is more, the link between these axioms and the notion of quality remained very obscure. In an article published in the Revue de Métaphysique et de Morale in 1899 (see [Poincaré, 1899]), Poincaré fiercely attacked this part of Russell's book. The French mathematician rightly pointed out that Russell's alleged axiomatization was mathematically empty. He also remarked that the (at the time) incipient topology was, more than the projective theory, entitled to be called a geometry of quality, since the qualitative invariance was built in the very definition of the topological transformations ${ }^{30}$.

Only two things were clear in the approach developed by Russell: his criticism of the analytical geometry and the importance he gave to von Staudt's quadrilateral construction (and, more generally, to the incidence relations between points, lines and planes ${ }^{31}$ ). Russell, however, neither gave the axioms of incidence which would allow one to prove the unicity of von Staudt's construction, nor explained the link between incidence relations and the notion of quality. The judgement of Dieudonné that I quoted in the introduction, even if harsh, was thus not mistaken. In its first version, Russell's theory of geometry was not convincing at all.

A new conception is exposed, two years later, in Russell's answer (written in French) to Poincaré's critical article ([Russell, 1899b]). In it, Russell completely gave up his notion of 'qualitative' geometry and delineated the nature of projective geometry more precisely. $\mathrm{He}$ acknowledged the relevance of Poincaré's criticism, and accepted the challenge in exposing a new axiomatic. I list the six postulates of Russell's new system ${ }^{32}$ :

Axiom I. There is a class $A$ of objects (...) such that any two of these objects, e. g. $A_{1}, A_{2}$, uniquely determine another object ( $a_{12}$ say) belonging to a different class $a$. (...) If the object $a_{12}$ determined by $A_{1}$ and $A_{2}$ is not identical with the object $a_{13}$ determined by $A_{1}$ and $A_{3}$, then the three objects $A_{1}, A_{2}, A_{3}$ uniquely determine an object $\left(\alpha_{123}\right)$ belonging to a new class $\alpha$, which again does not determine uniquely the objects from which it is determined. Moreover $a_{12}$ and $\alpha_{123}$ are independent of the order of the determining objects; and $\alpha_{123}$ is also determined by $A_{1}$ and $a_{23}$, or by $A_{2}$ and $a_{31}$, or by $A_{3}$ and $a_{12}$.

Axiom II. Two objects of class $\alpha\left(\alpha_{1}\right.$ and $\left.\alpha_{2}\right)$ determine uniquely an object ${ }_{12} a$ of class $a$; and if ${ }_{12} a$ is not identical with ${ }_{13} a$, then $\alpha_{1}$ and $\alpha_{2}$ and $\alpha_{3}$ determine uniquely an object ${ }_{123} A$ of class $A$, which is also determined by $\alpha_{1}$ and ${ }_{23} a$. Two objects of class $a$, or four of class $A$ or of class $\alpha$, determine nothing. Thus all the objects determined by means of objects in the classes $A, a, \alpha$ belong in turn to these three classes.

Axiom III. When two objects $\alpha_{123}, \alpha_{124}$ are respectively determined by $A_{1}, A_{2}, A_{3}$

\footnotetext{
${ }^{29}$ The three alleged 'postulates' were: 'I We can distinguish different parts of space, but all are qualitatively similar, and are distinguished only by the immediate fact that they lie outside one another. II Space is continuous and infinitely divisible; the result of the infinite division, the zero of extension, is called a point. III Any two points determine a unique figure, called a straight line, any three in general determine a unique figure, the plane.' ([Russell, 1897], p. 133).

${ }^{30}$ Poincaré developed this idea in his [Poincaré, 1902] (see p. 60).

${ }^{31}$ For instance, Russell explained that 'the successive application, to any figure, of two reciprocal operations of projection and section, is regarded as producing a figure projectively indistinguishable from the first (...)' ([Russell, 1897], p. 123) and that '[the quadrilateral construction] proceeds entirely by means of the general principles of [projective] transformation' ([Russell, 1897], p. 127)

${ }^{32}$ There is an extended English version of Russell's published article ([Russell, 1899a]). The manuscript has been published in the volume 2 of the Bertrand Russell Collected Papers. I quote here the English manuscript.
} 
and by $A_{1}, A_{2}, A_{4}$, the object of class $a$ determined by $\alpha_{123}$ and $\alpha_{124}$ is the same as that determined by $A_{1}$ and $A_{2}$.

Axiom IV. Three objects $\alpha_{123}, \alpha_{124}, \alpha_{125}$, determined respectively by $A_{1}, A_{2}, A_{3}$; $A_{1}, A_{2}, A_{4} ; A_{1}, A_{2}, A_{5}$, collectively determine nothing. Three objects $\alpha_{123}, \alpha_{145}$, $\alpha_{167}$ (provided the first and the second do not determine the same object $a$ as the first and third) collectively determine the object $A_{1}$.

Axiom V. Let $a_{23}$ be the object determined by $A_{2}$ and $A_{3}$, and $A$ the object determined by $a_{23}$ and $\alpha_{145}$. Then the object $a$ determined by $\alpha_{123}$ and $\alpha_{145}$ is the same as that determined by $A_{1}$ and $A_{2}$.

Axiom VI. When two objects ${ }_{123} A,{ }_{124} A$ are respectively determined by $\alpha_{1}, \alpha_{2}, \alpha_{3}$, and $\alpha_{1}, \alpha_{2}, \alpha_{4}$, they determine together the same object ${ }_{12} a$ as is determined by $\alpha_{1}$ and $\alpha_{2}$. ([Russell, 1899a], p. 403-404)

Russell also gave what he called an algebraic version of this system (Ibid., p. 404-405). Instead of containing three classes of signs, it involved only one type of symbols and a product operation. For instance if 1,2, 3 belonged to the class $\alpha$, the products 12, 13, 23 belonged to the class $a$, and 123 to the class $A$.

To understand the idea behind Russell's reasoning, one must interpret the classes $A, a, \alpha$ as respectively, the sets of planes, lines and points (or, dualy, as the sets of points, lines and planes), and interpret the product between these objects as the operation of projecting (two points, three points, one line and a point) or as the operation of sectioning (two planes, three planes, one line and a plane). Viewed in this way, the six postulates appear to put some conditions on the incidence relations between the three kinds of items ${ }^{33}$. And Russell succeeded in showing that these conditions suffice in order to deduce von Staudt's unicity theorem ${ }^{34}$.

Russell's system was in fact an elaboration from Grassmann's theory of progressive and regressive products. Russell learnt the doctrine through Whitehead's Universal Algebra, and the peculiarity of Russell's presentation can be explained by this genealogy. I have analysed in detail the relation between [Whitehead, 1898] and [Russell, 1899b] elsewhere ${ }^{35}$. But the following quotation of Whitehead's book should suffice, I think, to show the relevance of this comparing:

If $\rho+\sigma<v$, then $P_{\rho} P_{\sigma}$ is progressive and represents the containing region (..) of the two regions $P_{\rho}$ and $P_{\sigma}$; unless $P_{\rho}$ and $P_{\sigma}$ overlap, and in this case the progressive product $P_{\rho} P_{\sigma}$ is zero.

If $\rho+\sigma>v$, then $P_{\rho} P_{\sigma}$ is regressive and represents the complete region (...) common both to $P_{\rho}$ and $P_{\sigma}$; unless $P_{\rho}$ and $P_{\sigma}$ overlap in a region of order greater than $\rho+\sigma-v$, and in this case $P_{\rho} P_{\sigma}$ is zero.

If $\rho+\sigma=v$, then $\left(P_{\rho} P_{\sigma}\right)$ is a mere number and can be considered either as progressive or regressive. ([Whitehead, 1898], p. 191).

The progressive product was here introduced as an algebraic expression of the geometrical operation of 'projecting', the regressive product constituted an algebraic definition of the geometrical relation of 'sectioning'. The strange role played by the product in Russell's system finds here an explanation. For a reader of Whitehead's book, it was natural to connect the multiplication with the operations of 'projecting' and 'sectioning'. Note that Russell explicitly acknowledged Whitehead's influence in a letter to Couturat ${ }^{36}$.

\footnotetext{
${ }^{33}$ The first axiom explains how to connect the points to define a line, to connect three non collinear points, or to connect a line and a point, to define a plane. The second axiom stipulates that two planes determine a line, that three non coaxial planes, or one plane and one line not belonging to the plane, determine a point. The third axiom said that two planes determined by the points $A_{1}, A_{2}$ and $A_{3}$, and by the points $A_{1}, A_{2}$ and $A_{4}$, define the line $A_{1} A_{2}$. I let the reader interpret the others postulates

${ }^{34}$ With the help of the algebraic version of his postulates, Russell demonstates that if $1,2,3,4,5$ be five terms such that no two pairs and no two triads have the same product, then the expression $45\{23.4(12.345)(13.245)\}$ is constant so long as 145, 245, 345 are constant, however the terms 1, 2, 3, 4, 5 may be changed.' ([Russell, 1899a], p. 407); this, Russell said, amounts to prove von Staudt's unicity theorem.

${ }^{35}$ [Gandon, 2004].

${ }^{36}$ The letter dated from June the $21^{\text {th }}$ of 1900 . Russell went back over the enthusiastic review that his French
} 
In 1899, Russell, awoken by Poincaré, acknowledged the fact that its former characterisation of projective geometry as a science of quality had absolutely no mathematical meaning. But the philosopher remained opposed to the 'metrical' approach, according to which space was nothing other than a numerical manifold. The new idea was to focus only on the role played by the incidence relations between the various geometrical entities, and to rigorously deduce from them von Staudt's quadrilateral construction geometry. In this second stage of the evolution of Russell's thought, everything happens as if giving a philosophical meaning to the projective approach of metageometry consisted of displaying the true form of incidence relations ${ }^{37}$.

However, a difficulty still burdened Russell's conception. As we have said above, Klein had clearly shown that von Staudt's reasoning could not give what it claimed to give, and needed to be expanded. Now, in his [Russell, 1899b], Russell claimed that (p. 405):

To show that [the above] axioms suffice, it is only necessary to prove (...) the uniqueness of von Staudt's quadrilateral construction, since all projective geometry (...) follows from this construction.

Did Russell ignore the discovery of Klein? No. In another passage, Russell added:

There is only one proposition of importance which this method, so far as I know, is incapable of proving. This is the proposition that all points of a line can be obtained by this construction, and that there is no finite gap in the line.

The mention of the 'finite gaps' strongly recalls Klein's criticism of von Staudt's proofs. But then, if von Staudt's procedure is acknowledged as not being powerful enough, what are we supposed to do with the projective theorems that are not deducible by a reasoning à la von Staudt? Russell remained very vague on this crucial issue. In Klein's wake, he seemed to believe that some ordinal considerations were required to complete von Staudt's programme. But he was very hesitant about how to deal with order. Was order a purely projective concept, or was it a metrical notion? Russell did not answer that crucial question ${ }^{38}$, and this cast a shadow over his general theory of geometry. A central problem was left unresolved: how could one reconcile the idea that projective geometry should be separated from any metrical consideration, with the fact that von Staudt's approach, based on the sole incidence relations, cannot provide it with an adequate foundation? How could we rescue von Staudt's programme without renouncing the idea that projective geometry is independent from any metrical considerations?

The discovery of Pieri's work allowed Russell to remove all the strains put on his analysis. Pieri succeeded in achieving von Staudt's programme ${ }^{39}$. As we have said, he managed to deduce the fundamental theorem (and thus the whole body of projective geometry), without introducing new ordinal indefinables.

friend devoted to Whitehead's book: 'I have liked very much your review of Whitehead's book, and Whitehead felt the same. However, I would like to point out what it seems to me is a mistake. Grassmann's algebra (even before the multiplication) is not the one of the completely pure projective geometry: intensity has in it an essential role; and, since it does not represent a mass, it could represent only a metrical idea. This is the reason why Whitehead, who agrees with me on this, speak not of projective geometry, but of descriptive geometry. You will see that it proves V. Staudt's construction without the third dimension (p. 215) - it could not do that by a genuine projective method. The true algebra of projective geometry is the one I have devised for my answer to Poincaré: it contains only the multiplication. I have convinced Whitehead of this fact, and he will put this algebra in his second volume.'

${ }^{37}$ Note that Poincaré, in an article dated from 1898, acknowledged that the 'géométrie de Staudt' provided an alternative to his own group theoretical foundation ([Poincaré, 2002], p. 28-29). He thought, however, that this path, based on the incidence relations, was very artificial: 'To obtain the notion of length only as a particular case of the anharmonic ratio is an artificial detour one is reluctant to make'.

${ }^{38}$ We found both concepts in Russell's writing at the time. In [Russell, 1899b] (especially p. 379-380: 'Projective Geometry is not essentially concerned with order or series.'), Russell seemed to consider that order was a metrical concept, and that pure projective geometry should be restricted to only a part of what was usually regarded as belonging to projective geometry (the fundamental theorem and Pappus's theorem would then be metrical...). At some other times (for instance in [Russell, 1898]), Russell seemed to regard order as a mere projective notion, without explaining how to connect it with incidence relations.

${ }^{39}$ Pieri actually translated von Staudt's Geometrie der Lage in Italian. For a study of the strong link between Von Staudt and Pieri, see [Marchisotto, 2006]. 
Let me quote a passage of [Russell, 1903] (p. 421), in which Russell summed up the story:

The true founder of non-quantitative Geometry is von Staudt. It was he who introduced the definition of a harmonic range by means of the quadrilateral construction, and who rendered it possible, by repetitions of this construction, to give projective definitions of all rational anharmonic ratios. [...] But there remained one further step, before projective Geometry could be considered complete, and this step was taken by Pieri. In Klein's account, it remains doubtful whether all sets of four collinear points have an anharmonic ratio, and whether any meaning can be assigned to irrational anharmonic ratios. For this purpose, we require a method of generating order among all the points of a line. For, if there be no order but that obtained from Klein's method, $[\ldots]$ there will be no way of assigning irrational coordinates to the points which do not have rational coordinates. There is, of course, no projective reason for supposing that there are such points; but there are metrical reasons, and in any case it is well, if possible, to be able to deal projectively with a continuous space. This is effected by Pieri, with the help of certain new axioms, but without any new indefinables. Thus at last the long process by which projective Geometry has purified itself from every metrical taint is completed. ([Russell, 1903], p. 421)

Russell still placed von Staudt's quadrilateral construction (i.e. the incidence relations) at the center of the projective world. But henceforth, he no longer had any problem with Klein's discovery: Pieri had 'completed' the edifice begun by G. von Staudt. In Russell's mature theory, thanks to Pieri's work, the severe difficulty which burdened his 1899 approach no longer existed. Pieri gave Russell the mathematical theory called for by his philosophy of geometry.

Since the beginning of his career, Russell represented himself as a follower of the pure 'synthetic' geometrical tradition. His problem lay in precisely delineating what the indefinables of pure geometry were. Pieri's work gave him the opportunity to define pure geometry as a theory of incidence relations. Russell's characterization of space as a 'multiple series' in chapter 54 is thus the upshot of the long evolution I have been describing: it allies his philosophical commitment to the pure synthetic tradition with Pieri's technical achievement. Furthemore, the story we have just told allows us to rejoin the puzzle raised at the end of the preceding section. Let us remind ourselves that Russell, while having the means to derive projective geometry from ordinal considerations, did not attach any importance to this formal approach - a position that seemed to us surprising, given the importance Russell attached to order in his logicist conception of mathematics. How could one explain Russell's preference for Pieri? Focusing on the global features of his logicism, Russell's theory of geometry could indeed appear to be needlessly complicated: to derive geometry from the relation 'between' would have well fitted Part IV and Part V of Principles, which are devoted to mathematical analysis. However, when Russell's involvement in the tradition of pure geometry is taken into account, its preference for Pieri's approach seems to be very natural: following Pasch would have been bringing geometry too close to analysis, and would have lead to renouncing the pure topic-specific conception of von Staudt. In order to explain Russell's taste, we thus have to take into account his 'local' analysis of what geometry was - his idea that geometry should not be reduced to coordinate geometry.

\section{Russell's theory and mathematical practices.}

Let me come back to Detlefsen's claim that Russell's logicism was an endeavour to reduce topicspecific mathematical knowledge to topic-neutral logical knowledge. Now, the upshot of my reading of chapters 54-56 of [Russell, 1903] seems to directly contradict this assertion. Russell did elaborate a topic-specific theory of geometry - he did try to delineate the outline of what makes geometry specific. Moreover, his quite sophisticated answer (geometry is at minima a theory of incidence) was based on a well identified mathematical programme (the pure synthetic geometry à la von Staudt), and on some (at the time) very recent advances within this tradition (Pieri's work). Finally, while Russell developed the technical machinery to develop the projective geometry from the concept of order, he purposely rejected the solution. This latter fact is especially worth emphasizing. Russell's official global approach should have led him to choose 
Pasch's way of presenting projective geometry; only local facts drove him to regard order as a derived concept and to adopt Pieri's approach.

However, even if all these elements tend to weaken Detlefsen's reading of Russell's standpoint, the account offered in Part VI of the Principles remains quite abstract. Russell gave two axiomatizations of projective geometry, and chose one of the two. Now, Detlefsen and Poincare never disputed the fact that geometry could be axiomatized - they only said that the knowledge the working geometers had did not coincide with the knowledge of the axiomatizers. Moreover, the fact that Russell was involved in a movement which aimed to defend the purity of geometrical knowledge does not in itself suffice to prove that his conception of geometry as a doctrine of incidence fitted the contemporary actual mathematical practices. Russell could have mistakenly believed this was the case.

In order to back up Russell's position, we have then to examine the relations between his definition of geometry and the works of the geometers of that period. Do Russell's axioms after he read Pieri actually support rigorous proofs of the kind that mathematicians at the time actually gave? Does the emphasis on incidence relations correspond to some features in contemporary geometrical practices? I will answer this question positively. I will firstly remark that the fundamental theorem of the projective geometry occupied a central position in the geometrical researche at the time. I will secondly show that Russell's emphasis on incidence relations was a common feature of all geometrical investigations based on the notion of finite configurations. I will thirdly focus on a very interesting paper of Veblen, alas unnoticed at the time, in which the mathematician extended Pieri's definition of order.

Peano's axiomatic of whole numbers, which was often taken by Russell himself as a paradigm of what an axiomatic system was, had no obvious connection with the works done in the theory of numbers at the turn of the twentieth Century. Peano's formal definition satisfied a purely foundational need, which had nothing to do with the contemporay arithmetical researche. Did the axiomatic approaches in geometry also come from some very peculiar requirements, which had nothing to do with the needs of the working geometers? This was definitely not the case. The axiomatization of the real projective geometry was closely connected to the issues concerning the proof of the fundamental theorem, which was then one of the main open problems. Let us recall that Klein showed the demonstration of v. Staudt was flawed; in Klein's wake, all the best geometers attempted to determine what were the necessary and sufficient conditions for proving the fundamental theorem. This question was thus on the agenda of Klein, Zeuthen, Darboux, Pieri, Enriques - but Wiener, Schur, Hilbert and Hölder also devoted some works to it ${ }^{40}$. The discussion of the various proofs of the fundamental theorem had then a structuring effect on geometry as a whole, in that it compelled mathematicians to size up the role of the different theorems of projective theory. There was then a close connection between the axiomatization of projective geometry and the actual geometrical works.

Concerning the fundamental theorem, two lines of research could be distinguished. The first one followed Klein's initial intuition - the idea was to reprise v. Staudt's proof by adding some ordinal axioms to the incidence postulates. This was the path taken in Zeuthen's, Darboux's and Enriques's works. But a new direction arose, at the beginning of the 1890's, with an important paper from the German mathematician H. Wiener. He asserted that a version of the fundamental theorem could be proved using the theorem of Pappus (i.e. incidence relations alone), without using continuity ([Wiener, 1891] $)^{41}$. F. Schur first showed in 1899, using some congruence axioms, that this was indeed the case ([Schur, 1899], [Schur, 1902]). These works, which aimed to prove the fundamental theorem without any continuity assumption, led to a strengthening of the link between geomety and the algebraic theory of fields. Let us recall that v. Staudt had developped a geometrical algebra of 'throw' - using the properties of projective involutions, the German mathematician succeeded in defining an addition and a product between points on the projective

\footnotetext{
${ }^{40} \mathrm{On}$ the history of the fundamental theorem of projective geometry, see [Voelke, 00] and [Nabonnand, 2006].

${ }^{41}$ In September 1891, Hilbert attended a lecture by Wiener on the foundations of geometry. Toepell said that this lecture inspired Hilbert to explore the use of the axiomatic approach to adress the problem raised by the projective treatment of the non euclidean geometry; see [Toepell, 1986].
} 
line ${ }^{42}$. Now F. Schur, extending some ideas of Hilbert, demonstrated in 1902 that there was an equivalence between the fundamental theorem of projective geometry and the commutativity of the product operation. In other words, the axioms of incidence given by Coxeter (see above section 2) allow us to introduce a division ring on the three dimensional space, but not a field (see [Coxeter, 1949]).

In all these works, Klein's considerations about the topology of the real projective line were replaced by a careful examination of the formal properties of some basic geometrical operations. The incidence relations and the various projective theorems were here used to define these operations (this was the case of Desargue's theorem) and to show that these operations had certain expected properties (this was the case of the fundamental theorem). I am not saying here that Russell had any influence on this line of thought, nor even that he knew about the existence of this deep and strong trend in research. I am just sustaining that the stress Russell put on the purity of method and on incidence relations fitted this geometrical practice well. If one does not find in Russell's writing any connection between geometrical constructions and field operations, the negative idea that one should not repair von Staudt's proof by introducing topological considerations is at the basis of both Wiener's and Russell's reaction. The very words Wiener used in his seminal article were actually pretty close to the terminology Russell used in his [Russell, 1899a] ${ }^{43}$. Of course, the philosopher did not announce, as Wiener did, that the fundamental theorem was a consequence of Pappus's theorem. But the background of both writers was akin, and this suffices to show that Russell's emphasis on incidence relations matched some mathematical leading interests.

Another thread, maybe stronger than the previous one, related Russell's theory to some contemporary geometrical works. I am referring here to the rise of the theory of configurations and of finite geometries. At the end of the twentieth Century, more and more mathematicians became aware of the numerous connections between combinatorial analysis and projective geometry. In his seminal article, E. H. Moore [Moore, 1896], elaborating on the work of Reye ([Reye, 1882]), made this link explicit, and developed the conceptual frame in which all future works would be conducted. Moore began his paper by giving the first general and abstract definition of the notion of configuration ${ }^{44}$ :

We have at disposal $n$ sets of objects (letters), $a_{1}$ in the first set, $a_{2}$ in the second set, and, in general, $a_{i}$ in the $i^{t h}$ set; we denote these objects by

$$
\lambda_{i j(i)},
$$

where the first suffix $[i=1,2, \ldots n]$ picks out the set to which the object belongs, and the second $\left[j^{(i)}=1,2, \ldots a a_{i}\right]$ picks out the object in that set.

\footnotetext{
${ }^{42}$ For a clear and brief presentation of Hessenberg's version of v. Staudt's calculus, see [Coxeter, 1947], p. 71-73. If $P_{1}, P_{2}, P_{\infty}$ are any three fixed collinear points, and $X, Y, Z$ three variables points on the same line, one defines the sum $X+Y$ as the point corresponding to $P_{0}$ in the involution denoted by the pairs $(X Y)$ and $\left(P_{\infty} P_{\infty}\right)$ and the product $X Y$ as the point corresponding to $P_{1}$ in the involution denoted by the pairs $(X Y)$ and $\left(P_{0} P_{\infty}\right)$. The associativity of addition and multiplication is a direct consequence of the axioms of incidence. The distributive law comes from the fact that, for any four collinear points $A, B, C$ and $D, A B C D$ is projectively related to $B A D C$.

${ }^{43}$ Let me quote the beginning of [Wiener, 1891] (p. 45-46): 'One can expect from a proof of a mathematical theorem that it uses only the assumptions from which the theorem actually depends. The minimal possible assumptions are the existence of certain objects and of certain operations by which the objects are linked together. [...] One instance of such a situation is provided bt the plane projective geometry. The objects are the points and the right lines - the operations are projecting and sectioning. Only a finite number of objects and operations are assumed to be given. Or, stripping off the geometrical clothes: let two kinds of elements and two kinds of operations be given, so that the connexion of two elements of the same kind give an element of the other kind.' As Wiener did so, Russell restricted himself to the operations of projection and section, and gave also a pure algebraic version of the postulates of incidence.

${ }^{44}$ Let me quote Moore: 'The technical term configuration was introduced - into projective geometry of the plane and of space - by Mr. Reye [...]. Particular geometric configurations, occuring in the plane, in space or, in general in flat space of $n$ dimensions, have been investigated by (among others) Mrs. De Vries, Jung, S. Kantor, Klein, Martinetti, Reye, Schoenfliess, Segre and Veronese. I have, however, come across no explicit formulation of the definition of the general geometrical configuration occurring in flat place of $n$ dimensions.'
} 
We have at disposal further a certain relation - call it incidence - between certain objects of different sets, so that we can say that $\lambda_{i_{1} j_{1}}$ is or is not incident with $\lambda_{i_{2} j_{2}}$ $\left(i_{1} \neq i_{2}\right)$. We tabulate these incidences occuring in the system of objects in a table of incidences of which one line is

$$
\lambda_{i_{1} j_{1}}\left[\lambda_{i_{1}^{\prime} j_{1}^{\prime}}, \lambda_{i_{2}^{\prime} j_{2}^{\prime}}, \ldots \lambda_{i_{k}^{\prime} j_{k}^{\prime}} \ldots\right]
$$

which is read, $\lambda_{i_{1} j_{1}}$ is incident with $\lambda_{i_{k}^{\prime} j_{k}^{\prime}}(k=1,2, \ldots)$.

This table of incidences, when arranged as to show also the distribution of the objects into the $n$ sets, furnishes the complete tactical definition of our system of $n$ sets of objects subject to the otherwise undefined incidence-relation.

This system is called a tactical configuration of rank $n$, if for every $g, h(g \neq h)$ every object of the set $g$ is incident with the same number, say $a_{g h}$, of objects of the set $h$. I inscribe the fundamental numbers $a_{i}, a_{g h}$ of a configuration of rank $n$ in the square matrix-symbol [with $a_{g g}=a_{g}$ ]

$$
\left(a_{g h}\right)
$$

which symbol serves likewise as the notation for the configuration.

To simplify let us rectrict ourselves to a configuration of rank $2(n=2)$; we have then two sets $a_{1}$ and $a_{2}$ of objects (for instance, the set of points and the set of lines), and the definition of a complete configuration amounts to defining a subset of $a_{1} \times a_{2}$ (this is still nowadays the general definition of an incidence structure, see [Dembowski, 1968]). Now, a configuration of rank 2 is called tactical if and only if every object of $a_{1}$ is incident with the same number of objects in $a_{2}$, and vice-versa. In this case, the configuration could be represented by a $2 \times 2$ square matrix $\left(\begin{array}{ll}a_{11} & a_{12} \\ a_{21} & a_{22}\end{array}\right)$, whose diagonal numbers $a_{11}$ and $a_{22}$ give the cardinality of $a_{1}$ and $a_{2}$, and of which the numbers $a_{12}$ and $a_{21}$ give respectively the number of elements of $a_{2}$ (the number of lines) on each element of $a_{1}$ (point) and the number of elements of $a_{1}$ (the number of points) on each element of $a_{2}$ (line). For instance, if $a_{1}$ is a set of coplanar projective points and $a_{2}$ a set of coplanar projective lines, Desargues's planar configuration can be noted $\left(\begin{array}{cc}10 & 3 \\ 3 & 10\end{array}\right)$ (or equivalently $\left.\left(10_{3}, 10_{3}\right)\right)$, since in such a configuration, we have ten points and ten lines such that each point is on three lines and each line passes by three points (see [Veblen and Young, 1910], p. 38-42). As examples of other geometrical configuration of rank 2, we could also mention $\left(9_{3}, 9_{3}\right)$ (one of these is Pappus's hexagon configuration), and $\left(12_{2}, 8_{3}\right)$. It is easily shown that a configuration of rank 2 is tactical only if $a_{11} a_{12}=a_{21} a_{22}$.

Note that if every geometrical planar configuration is not necessarily tactical, the converse is also true: some tactical configurations of rank 2 are not geometrically constructible. As Moore said, 'particular types of configurations are reached by particularizing the incidence-relations' ([Moore, 1896], p. 266) ${ }^{45}$. According to E. Steinitz and E. Merlin, the authors of the article entitled 'Configurations' in the French version of the Encyclopedia of the mathematical sciences, one of the central issues of these investigations was to formally characterise, among the possible tactical configurations, which one could be geometrically realizable (see [Molk, 1916], tome III, vol. 2, p. 149-152). For instance, $\left(7_{3}, 7_{3}\right)$ (the so called seven points model of the projective plane) is not geometrically constructible. According to the same mathematicians, another central question was to enumerate and to classify all the tactical configurations of a certain sort ${ }^{46}$. For instance, it was known that there were three distinct $9_{3}$ configurations and five $\left(12_{2}, 8_{3}\right)$ ones. The main motive behind this research seems, however, to have been to give a general and unified

\footnotetext{
${ }^{45}$ In a configuration of rank 2 , it is not necessarily the case that, if an element of $a_{1}$ is incident with an element of $a_{2}$, the latter must be incident with the former - this condition, which is obviously necessary to have a geometrically constructible configuration, is not contained in the general definition of the tactical configuration. This shows how general Moore's point of view is.

${ }^{46}$ See [Molk, 1916].
} 
framework for dealing with some purely combinatorial questions, such as Kirkman's fifteenschoolgirls problem ${ }^{47}$. In 1906, a new stage was reached with the article of O. Veblen and W. H. Bussey on finite projective geometries [Veblen and Bussey, 1906]. The two authors used the axiomatic approach ${ }^{48}$ to generate a class of tactical configurations 'which includes many well known configurations as well as many new ones' (p. 241). In brief, the configurations was seen as some 'models' of the projective axioms, and, in return, projective tools were used to study the formal properties of the tactical configurations. Geometrical configurations were thus no longer conceived as a kind of constructible figure embedded in an already given space - a configuration which satisfied the projective axioms was now regarded as being itself a projective space. Note that Veblen's and Bussey's researche were not completely severed from the issues surrounding the fundamental theorem. Elaborating on a previous work on finite algebras done by J. H. Maclagan-Wedderburn, the two mathematicians showed that Pappus's theorem was true in all finite projective geometries of dimension strictly greater than two, without further hypothesis ${ }^{49}$.

I did not find any thorough historical study devoted specifically to the theory of configurations. [Dembowski, 1968] and [Buekenhout, 1995] provide many useful insights, but they primarily focus on the developments of incidence theory in the twentieth Century, especially in connection with the post-Fisherian statistical theory of experimental design ${ }^{50}$. The chapter on 'Configuration' contained in Molk's Encyclopedia could have been a starting point, but, unfortunately, it was left unfinished because of the First World War ${ }^{51}$. Given the mathematical wealth of these works, one can only regret this historiographical lack, that my few preceding remarks cannot even begin to fill. However, they seem to be sufficiently convergent to indicate that, at the turn of the twentieth Century, a kind of crystallization of until then various mathematical practices (geometry, combinatorics, enumeration, etc...) based on the abstractly defined notion of incidence, took place. It seems indeed, that, after Moore, all the mathematicians working on the configurations based their argument on the technical notion of incidence. They all used some very specific notation (as for instance matrix or tabular notation) and they all employed some devices coming from finite algebra to investigate the formal properties of the finite incidence structures. Moreover, all these works referred to each other and formed a mathematical 'genre'.

Was there a link between Russell's theory of geometry and the works on incidence structure? Russell's definition of geometry in terms of incidence fits the general features of the mathematical movement just described very well. Again, I am not claiming that Russell was aware of this connection. In [Russell, 1903], nowhere did he allude either to the theory of configuration or to finite geometries. But this lack of explicit link should not make the conceptual ties which connect Russell's conceptions with these ongoing mathematical works disappear. The theory of configuration undoubtely provides Russell's philosophical views with a mathematical echo.

Moreover, if Russell did not speak about the works of Moore, Veblen and so on, Whitehead, in his tract on projective geometry [Whitehead, 1906], which is presented by him as an extension of [Russell, 1903] Part VI, gave them a central place. In his preface, Whitehead defined geometry as 'the science of cross-classification' (cross-classification is Whitehead's name for in-

\footnotetext{
${ }^{47}$ Kirkman's problem is arranging fifteen school girls in parties of three for seven consecutive days' walk so that every two of the girls walk together once and only once during the seven days. More generally, an arrangement of $m$ elements in sets of three so that every set of two is contained in one and only one set of three, is called a triple system of $m$ elements. De Vries showed in 1894 that $m$ every triple system may be looked at as a configuration $\left(\begin{array}{cc}m & \frac{1}{2}(m-1) \\ 3 & \frac{1}{6} m(m-1)\end{array}\right)$. For instance, the solution of Kirkman's problem is given by the configuration $\left(15_{7}, 35_{3}\right)$.

${ }^{48}$ The axiomatic of projective geometry presented in [Veblen and Bussey, 1906] contains only incidence axioms.

${ }^{49}$ See [Veblen and Bussey, 1906], p. 246. Note that this did not amount to prove that the fundamental theorem of the projective geometry was valid in every finite geometry. Veblen and Bussey showed how to define a finite projective space from a Galois field $G F\left(p^{n}\right)$, with $p$ prime. If $n=1$, and if the dimension of the projective space is strictly greater than 2 , then the fundamental theorem holds, without further ado. But this is not necessarily the case, if $n>1$. On this, see [Veblen and Bussey, 1906], p. 248-249.

${ }^{50}$ As Dembowski explained in his introduction, the main reason to study finite geometry in the fourties or fifties came from statistics - the idea that an experiment should be carefully devised and organized led to expounding the 'right way' to arrange the experimental units into groups (blocks) so as to reduce irrelevant sources of variation between units.

${ }^{51}$ However, the chapter contains a section on the history of the notion of configuration and many bibliographical references.
} 
cidence). His definition was explicitly motivated by a reference to 'the modern Geometries with finite numbers of points'. Furthemore, in the book, Whitehead referred many times to the works of Veblen and Maclagan-Wedderburn ${ }^{52}$. A connection between Russell's conception of geometry on [Russell, 1903] and the works on finite geometries was then made by Whitehead as early as 1906.

I would like to add a last remark, focusing more on the role Russell gave to Pieri's work. In his book on real algebra [Sinaceur, 1991], H. Sinaceur explained that Pieri's idea to define order in terms of incidence relations, foreshadowed the great mathematical works of Artin and Schreier, developed only in the thirties. Let me summarize the content of Sinaceur's analysis.

In 1906, Veblen published a very brief article (three pages), entitled The square root and the relation of order, of which the explicit purpose was to 'infer order relations in terms of postulates about a square root'. Let me quote the beginning of Veblen's paper:

One of the most obvious discriminations between positive and negative numbers is that the former possesses square roots in the field of reals while the latter does not. This distinction, however, has not yet been used in any of the current systems of postulates. On the contrary, an order relation, $<$, is usually introduced as an undefined symbol.

Through the existence of a square root cannot be deduced from order relations without the use of a continuity assumption of some sort, it turns out to be very easy to infer the order relations from postulates about the existence of a square root. Suppose we are given a field [...]. A mark, $a$, is called a square if there exists a mark, $x$, such that $x x=a$. If not a square, a mark is called a not-square. Now add the postulates:

a) The marks -1 is a not-square

$\beta$ ) If marks $x$ and $y$ are not-square, then $x+y$ is a not-square. (p. 197)

Having deduced from that some theorems ${ }^{53}$, Veblen concluded:

It is now evident, on comparison, for instance with Huntington's set of postulates for real algebra [...] that our field satisfies all the conditions of the real number system except that of continuity. We therefore suggest as an elegant way of stating the postulates for the real number system in terms of the undefined symbols, + and $\times$, a set of postulates for a field in general, postulates $\alpha$ ) and $\beta$ ), and a continuity postulate [that could be defined without referring to an undefined ordinal relation].

Veblen succeeded then in giving a definition of the real field in a purely abstract and algebraic manner, without using any undefined order relations.

Now, as H. Sinaceur observed, the idea of deriving order from the distinction between square and not-square elements anticipated the construction of the purely algebraic theory of the real field by Artin and Schreier in [Artin and Schreier, 1926]. The core of their construction was precisely defining the real field by the condition that -1 cannot be written as a sum of square numbers, that is, by the very condition used in Veblen's article. Thus, as H. Sinaceur says ([Sinaceur, 1991], p. 229), 'one could think that there is a relation between these three pages from Veblen and [Artin and Schreier, 1926]'. Through she adds: 'the reality is very different, however! B. L. van der Waerden, who took part in the seminar sessions where the theory of the real fields was developed, thinks that neither Artin nor Schreier knew of this short article from Veblen'; and she concludes: 'Between Veblen's brainwave and [Artin and Schreier, 1926], the relation is conceptual, not historical. This makes it even more noteworthy'.

What has all that to do with Russell? Veblen concluded his article by a very precise reference to Pieri:

\footnotetext{
${ }^{52}$ I have analysed in detail the definition of geometry given by Whitehead in [Gandon, 2004].

${ }^{53}$ Veblen defined $a>b$ as meaning that $a-b$ is a square which is not zero, and $a<b$ as meaning that $a-b$ is a not-square; he proved then that the relation $>$ is connected (if $a \neq b$, then $a<b$ or $a>b$ ), transitive, dense, and compatible with addition and product.
} 
What may be thought of as the geometric analogue of the above determination of order relations has been carried through by M. Pieri on page 24 of his paper, I Principii della Geometria di Posizione [...]. Two point-pairs, $A B$ and $C D$, are said not to separate, or to separate, according as there exist, or do not exist, two points $H, H^{\prime}$ which are harmonically conjugate both with respect to $A B$ and to $C D$. Determining the points $H H^{\prime}$ is equivalent to finding the double points of the involution in which $A B$ and $C D$ are pairs of conjugate points and is therefore a problem of the second degree.

Pieri's use of Darboux's lemma was then the source of Veblen's powerful idea. In fact, Veblen provided only an algebraic version of Pieri's definition of order. Now, Veblen's reading of Pieri's work here was exactly the same as Russell's. For the two thinkers, the true import of Pieri's axiomatic was the way order was derived from Darboux's lemma. Veblen, as a mathematician, attempted to use this device to give a pure algebraic definition of the real field. Russell recognized in this move the (until then) missing ingredient, which prevented the completion of a pure theory of projective geometry. The two reactions were very different, but they came from exactly the same understanding of Pieri's work. Russell then, shares with Veblen the merit of having been the only one to grasp (each in their own specific style) the depth of Pieri's ideas.

Russell's views on geometry, especially his emphasis on the importance of incidence, was in 1903 not cut off from the contemporary mathematical practices. Firstly, the issues surrounding the fundamental theorem were central at the time, and Russell's distrust of ordinal considerations places his reasoning in a determinate current of research. Secondly, at the end of the XIXth Century, some geometers developed what they called a theory of configuration, which was nothing more than an abstract theory of incidence structures. At last, Veblen's article, based on Pieri's definition of order in terms of incidence, anticipated the development of Artin's and Schreier's works on real closed algebra. Of course, I am not claiming that Russell was aware of all these works, just that his idea that incidence relations lay at the heart of geometry was not mathematically empty. Note that I am not saying either that the movements we just discussed above were prevailing at the time. Klein's Erlanger programme, for instance, could well be regarded as the real unifying approach, and, seen from this perspective, Poincaré's emphasis on the role of group theory in geometry could be preferred to Russell's conception ${ }^{54}$. Russell's decision to define geometry as a theory of incidence was perhaps historically inaccurate. The philosopher could legitimely be criticized for having exaggerated the importance of what was then just a branch line in the development of the science. My point is however that Russell's definition of geometry corresponded to a trail which was then followed. This is enough to support that the opposition between Russell and Poincaré should not be seen as an opposition between a topicneutral, abstract and technically empty philosophical approach, and a topic-specific, concrete and informed conception of geometry, but rather as an opposition between two ways of viewing what made geometry specific at the time.

\section{Conclusion: toward a topic-specific logicism?}

In a way, every reductionist program is liable to be a target for the kind of criticism Detlefsen has adressed to Russell. Since, by definition, a reduction is a transformation of a given subject into another one, one can never be sure that, in making such a move, an essential feature of the initial subject is not lost. To reduce is to translate, and 'traduttore, traditore'. In mathematics, this issue is particulary pressing, since the distinction between the substantial content and the accidental form of any given mathematical theorem, or of any given proof, is a notoriously vexing question. To come back to one of our earlier examples, one can prove that, given some reasonable hypothesis, the fundamental theorem of projective geometry is equivalent to the commutativity of the product operation of v. Staudt's 'throw' algebra. No one, however, would claim that this change in the formulation is a mathematically unimportant fact. In mathematics more than

\footnotetext{
${ }^{54}$ For an elucidation of the connection between Poincaré's talks of intuition and his mathematical works, see [McLarty, 1997].
} 
anywhere else, the reductionist approach is then in danger of missing the substantial import of what is supposed to be reduced. Detlefsen's distinction between topic-specific and topic-neutral knowledge has thus the virtue of indicating a very deep problem which threathens every kind of reductionism in mathematics.

The purpose of my study of The Principles, part VI, was to show that the historical Russell did not ressemble the person portrayed by Detlefsen: Russell's choice for Pieri's account of projective geometry displayed a very high 'sensitivity to a grasp of local architecture'. The philosopher had indeed all the reasons in the world for choosing Pasch rather than Pieri. Pasch's emphasis on order fitted perfectly well one of the main feature of Russell's point of view, according to which order was the fundamental concept of mathematics (and a fundamental concept in metaphysics too; see the notorious Part IV of [Russell, 1903]). In a way, Russell's choice for Pieri was then a decision which went against the whole logicist tide. This is precisely what makes Russell's theory of geometry a very interesting case study. Geometry lay in the Principles at the junction of two different, and even in some ways, opposing contexts. On the one hand, Russell claimed that geometry belonged to pure mathematics, i.e. to logic; for him, geometry was nothing else than a part of the theory of relations and of set-theory. On the other hand, Russell saw himself as a follower of von Staudt; he was thus very anxious not to hide the specificity of projective geometry behind some foreign (possibly logical) 'hieroglyph'.

Now, Detlefesen's demand to have a topic-specific approach of the mathematical subject investigated did not go in the same direction when inserted into these two contexts. If the logicist reductionist stance seems, at first glance, to go along with a topic-neutral conception of geometry, then the endorsement of the synthetic point of view militates in favour of a topicspecific approach of geometry.

Things are thus much more complicated than Detlefsen seemed to believe. To develop a logicist theory of geometry was not, for Russell, a question of deducing all geometrical theorems within some predetermined standardized canvas: it was about delineating what makes all these theorems geometrical. For Russell, logic was not simply the means by which to get rid of any geometrical intuition - it was also a means of unfolding the logical shape of what Detlefsen's Poincaré called mathematical intuition.

Russell wanted then to have both a topic-specific theory of geometry and an overall logicist conception of mathematics. But how could he achieve that? The issue is henceforth to specify how it is possible (if it is) to reconcile the reductionist logicist stance with Russell's philosophy of geometry. Is a reductionist stance compatible with a defense of the specificities of the mathematical disciplines which are to be reduced? Would it be possible to define what we could call a 'topic-specific logicism'? And was Russell's conception close to this form of reductionism?

If these questions can appear rather strange to scholars accustomed to focusing exclusively on Russell's notorious claim about the whole numbers, let me stress that they arise very naturally to readers of the unduly neglected works that Russell devoted to projective geometry. And these questions are not only still open-ended ${ }^{55}$; they remain, today, very pressing. Indeed, nowadays, more and more philosophers stress the vital importance of developing a philosophy of mathematics which could account for actual mathematical practices ${ }^{56}$. In this context, the idea that logicism could be topic-specific may seem very attractive. Of course, since Russell's time, many things have changed in mathematics and in logic. But it seems to me that the philosophical idea of elaborating a logicism sensitive to the local design of the mathematical theories still represents an option worth pursuing.

\section{Acknowledgements}

This paper was first presented at the conference 'Qu'est ce que la géométrie aux époques modernes et contemporaines?' (16-20 April 2007), organized by the University of Köln and the

\footnotetext{
${ }^{55}$ In [Gandon, 2008b], I emphasized the role played by the notion of relational type in the Principles. But more works need to be carried out in order to expound a coherent picture of Russell logicism, when seen in light of his works in geometry.

${ }^{56}$ See for instance [Shapiro, 2000] (especially p. 143-180) and [Maddy, 2000] (especially p. 158-160); see, as well, the volume 15(3) of Philosophia Mathematica, where two papers ([Blanchette, 2007] and [Rav, 2007]) were devoted to the issue of topic-specificity.
} 
Archives Poincaré. I would like to thank Philippe Nabonnand for having enlightened me about the issues relative to projective geometry. I would like also to thank Nicholas Griffin, Brice Halimi, Bernard Linsky, Marco Panza, Ivahn Smadja for their helpful discussions. Many thanks also to the two anonymous referees for their useful suggestions.

\section{References}

[Artin and Schreier, 1926] Artin, E. and Schreier, O. (1926). Algebraische Konstruction reeller Körper. Abh. math. Sem. Hamb., 5:85-99.

[Blanchette, 2007] Blanchette, P. A. (2007). Frege on Consistency and Conceptual Analysis. Philosophia Mathematica, 15(3):321-346.

[Boolos, 1998] Boolos, G. (1998). The Advantages of Honest Toil over Theft. In Jeffrey, R., editor, Logic, Logic and Logic, pages 255-274. Harvard University Press.

[Buekenhout, 1995] Buekenhout, F., editor (1995). Handbook of Incidence Geometry. Elsevier.

[Carnot, 1803] Carnot, L. (1803). Géométrie de Position. Blanchard (repr.).

[Coxeter, 1947] Coxeter, H. S. M. (1947). Non-Euclidean Geometry. Toronto University Press, Toronto.

[Coxeter, 1949] Coxeter, H. S. M. (1949). The Real Projective Plane. Maple Press Company, York.

[Dembowski, 1968] Dembowski, P. (1968). Finite Geometries. Springer Verlag.

[Detlefsen, 1990] Detlefsen, M. (1990). Brouwerian intuitionism. Mind, 99:501-534.

[Detlefsen, 1993] Detlefsen, M. (1993). Poincaré vsus Russell on the Role of Logic in Mathematics. Philosophia Mathematica, 1:24-49.

[Dugac, 2003] Dugac, P. (2003). Histoire de l'Analyse. Autour de la notion de limite et de ses voisinages. Vuibert.

[Freudenthal, 1974] Freudenthal, H. (1974). The impact of von Staudt's foundations of geometry. In For Dirk Struik, pages 189-200. Dordrecht.

[Gandon, 2004] Gandon, S. (2004). Russell et l'Universal Algebra de Whitehead: la géométrie projective entre ordre et incidence (1898-1903). Revue d'Histoire des Mathématiques, 10:187256.

[Gandon, 2008a] Gandon, S. (2008a). La réception des Vorlesungen über neuere Geometrie de Pasch par Peano. Revue d'histoire des mathématiques.

[Gandon, 2008b] Gandon, S. (2008b). Which arithmeticisation for which logicism? Russell on relations and quantities in The Principles of Mathematics. History and Philosophy of Logic.

[Griffin, 1991] Griffin, N. (1991). Russell's Idealist Apprenticeship. Clarendon, Oxford.

[Heinzmann, 1986] Heinzmann, G., editor (1986). Poincaré, Russell, Zermelo et Peano. Blanchard, Paris.

[Hylton, 1990] Hylton, P. (1990). Russell, Idealism and the Emergence of Analytical Philosophy. Oxford University Press.

[Klein, 1871] Klein, C. F. (1871). Ueber die sogenannte nicht-euklidische geometrie. Mathematische Annalen, 4.

[Klein, 1873] Klein, C. F. (1873). Ueber die sogenannte nich-euklidische geometrie, zweiter aufsatz. Mathematische Annalen, 6. 
[Landini, 1998] Landini, G. (1998). Russell's Hidden Substitutional Theory. Oxford University Press, Oxford.

[Linsky, 1999] Linsky, B. (1999). Russell's Metaphysical Logic. CSLI Publications, Stanford.

[Maddy, 2000] Maddy, P. (2000). Naturalism in Mathematics. Oxford University Press.

[Marchisotto, 1995] Marchisotto, E. (1995). In the Shadows of Giants: The works of Mario Pieri in the foundations of mathematics. History and Philosophy of Logic, 16:107-119.

[Marchisotto, 2006] Marchisotto, E. (2006). The projective geometry of Mario Pieri: A legacy of Georg Karl Christian von Staudt. Historia Mathematica, 33:277-314.

[McLarty, 1997] McLarty, C. (1997). Poincaré: Mathematics \& Logic \& Intuition. Philosophia Mathematica, 5:97-115.

[Molk, 1916] Molk, J., editor (1906-1916). Encyclopédie des sciences mathématiques pures et appliquées. Gauthier-Villars, Paris, French edition.

[Moore, 1896] Moore, E. H. (1896). Tactical memoranda i-iii. American Journal of Mathematics, $18: 264-290$.

[Nabonnand, 2006] Nabonnand, P. (2006). Contributions à l'histoire de la géométrie projective au XIXème siècle. Thèse d'habilitation. Not yet published.

[Pasch, 1882] Pasch, M. (1882). Vorlesungen über neure Geometrie. Teubner, Leipzig.

[Peano, 1894] Peano, G. (1894). Sui fondamenti della geometria. Rivista di matematica, 4:51-90.

[Pieri, 1898] Pieri, M. (1898). I principii della geometria di posizione, composta in un sistema logico-deduttivo. Memoria della R. Accademia delle Scienze di Torino, 2:1-62.

[Poincaré, 1899] Poincaré, H. (1899). Des fondements de la géométrie: à propos d'un livre de M. Russell. Revue de Métaphysique et de Morale, 7:251-279.

[Poincaré, 1900] Poincaré, H. (1900). Sur les principes de la géométrie: réponse à m. russell. Revue de Métaphysique et de Morale, 8:73-86.

[Poincaré, 1902] Poincaré, H. (1902). La Science et l'Hypothèse. Flammarion, Paris, 1968 edition.

[Poincaré, 1906] Poincaré, H. (1905-1906). Les mathematiques et la logique (in 3 parts). Revue de Métaphysique et de Morale, 13-14:815-835; 17-34; 294-317.

[Poincaré, 1908] Poincaré, H. (1908). Science et méthode. Flammarion.

[Poincaré, 1909] Poincaré, H. (1909). La logique de l'infini. Revue de Métaphysique et de Morale, $17: 461-482$.

[Poincaré, 2002] Poincaré, H. (2002). Des fondements de la géométrie (1898). In Scientific Opportunism / L'opportunisme scientifique. Birkhäuser.

[Rav, 2007] Rav, Y. (2007). A Critique of a Formalist-Mechanist Version of the Justification of Arguments in Mathematicians' Proof Practices. Philosophia Mathematica, 15(3):291-320.

[Reye, 1882] Reye, T. (1882). Das Problem der Configuration. Acta Mathematica, 1:92-96.

[Richards, 1988] Richards, J. L. (1988). Mathematical Visions: The Pursuit of Geometry in Victorian England. Academic Press.

[Russell, 1897] Russell, B. (1897). An Essay on the Foundations of Geometry. Cambridge University Press. 
[Russell, 1898] Russell, B. (1898). Notes on Order. In Collected Papers, vol. II, pages 339-358. Routledge.

[Russell, 1899a] Russell, B. (1899a). The Axioms of Geometry, English version of Sur les axiomes de géométrie. In Collected Papers, vol. II, pages 352-362. Routledge.

[Russell, 1899b] Russell, B. (1899b). Sur les axiomes de la géométrie. Revue de Métaphysique et de Morale, 7:684-707.

[Russell, 1903] Russell, B. (1903). The Principles of Mathematics. Routledge, London.

[Russell, 1906] Russell, B. (1906). Les paradoxes de la logique. Revue de Métaphysique et de Morale, 14:627-650.

[Russell, 1910] Russell, B. (1910). La théorie des types logiques. Revue de Métaphysique et de Morale, 18:263-301.

[Russell, 1911] Russell, B. (1911). L'importance philosophique de la logistique. Revue de Métaphysique et de Morale, 19:282-291.

[Schur, 1899] Schur, F. (1899). Über den Fundamentalsatz der projectiven Geometrie. Mathematische Annalen, 51:401-409.

[Schur, 1902] Schur, F. (1902). Über die Grundlagen der Geometrie. Mathematischen Annalen, 55:265-292.

[Shapiro, 2000] Shapiro, S. (2000). Philosophy of Mathematics: Structure and Ontology. Oxford University Press.

[Sinaceur, 1991] Sinaceur, H. (1991). Corps et Modèles. Vrin, Paris.

[Toepell, 1986] Toepell, M. (1986). On the origins of David Hilbert's Grundlagen der Geometrie. Archive for History of Exact Sciences, 35:329-344.

[Toretti, 1978] Toretti, R. (1978). Philosophy of Geometry from Riemann to Poincaré. D. Reidel Publishing Company.

[Veblen and Bussey, 1906] Veblen, O. and Bussey, W. H. (1906). Finite projective geometries. Transactions ofthe American Mathematical Society, pages 241-259.

[Veblen and Young, 1910] Veblen, O. and Young, J. W. (1910). Projective Geometry, volume I. Ginn and Company.

[Voelke, 00] Voelke, J.-D. (00). Le théorème fondamental de la géométrie projective : évolution de sa preuve entre 1847 et 1900. Archive for History of Exact Science. Not yet published.

[Whitehead, 1898] Whitehead, A. N. (1898). A Treatrise on Universal Algebra With Applications. Cambridge University Press.

[Whitehead, 1906] Whitehead, A.-N. (1906). The Axioms of Projective Geometry. Cambridge University Press, Cambridge.

[Wiener, 1891] Wiener, H. (1890-1891). Über die Grundlagen und den Aufbau der Geometrie. Jahresbericht der Deutschen Mathematikervereinigung, 1:45-48. 